\section{BRAZIULIAN JOURNAL}

OF MEDICAL AND BIOLOGICAL RESFARCH

www.bjournal.com.br
ISSN 0100-879X

Volume 43 (5) 381-496 May 2011

BIOMEDICAL SCIENCES

AND

CLINICAL INVESTIGATION

Braz J Med Biol Res, May 2011, Volume 44(5) 483-488

doi: 10.1590/S0100-879X2011007500057

Effect of blocking Rac1 expression in cholangiocarcinoma QBC939 cells

Xudong Liu and Guangyi Wang

The Brazilian Journal of Medical and Biological Research is partially financed by

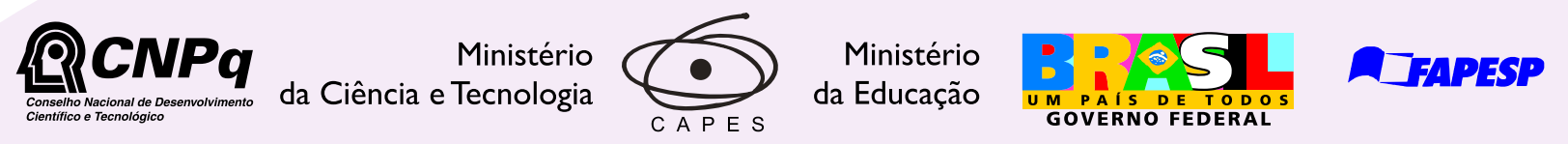

Institutional Sponsors

scie/
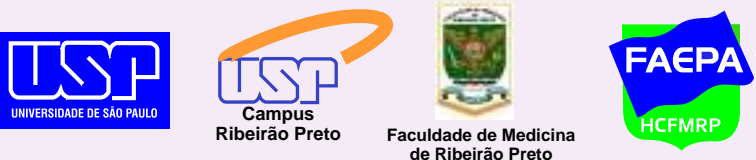

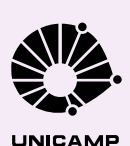

Ф SHIMADZU

GE Healthcare
Hotsite of proteomics metabolomics developped by:

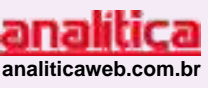

Thermo SCIENTIFIC 


\title{
Effect of blocking Rac1 expression in cholangiocarcinoma QBC939 cells
}

\author{
Xudong Liu ${ }^{1,2}$ and Guangyi Wang ${ }^{1}$
}

${ }^{1}$ Department of General Surgery, First Clinical Hospital, Jilin University, Changchun, Jilin, China ${ }^{2}$ Department of Emergency, Affiliated Hospital, Inner Mongolia Medical College, Hohhot, Inner Mongolia, China

\begin{abstract}
Cholangiocarcinomas (CCs) are malignant tumors that originate from epithelial cells lining the biliary tree and gallbladder. Ras correlative C3 creotoxin substrate 1 (Rac1), a small guanosine triphosphatase, is a critical mediator of various aspects of endothelial cell functions. The objective of the present investigation was to study the effect of blocking Rac1 expression in CCs. Seventy-four extrahepatic CC (ECC) specimens and matched adjacent normal mucosa were obtained from the Department of Pathology, Inner Mongolia Medicine Hospital, between 2007 and 2009. Our results showed that the expression of Rac1 was significantly higher $(53.12 \%)$ in tumor tissues than in normal tissues. Western blotting data indicated a significant reduction in Rac1-miRNA cell protein levels. Rac1-miRNA cell growth rate was significantly different at 24, 48, and $72 \mathrm{~h}$ after transfection. Flow cytometry analysis showed that Rac1-miRNA cells undergo apoptosis more effectively than control QBC939 cells. Blocking Rac1 expression by RNAi effectively inhibits the growth of CCs. miRNA silencing of the Rac1 gene suppresses proliferation and induces apoptosis of QBC939 cells. These results suggest that Rac1 may be a new gene therapy target for CC. Blocking Rac1 expression in CC cells induces apoptosis of these tumor cells and may thus represent a new therapeutic approach.
\end{abstract}

Key words: Cholangiocarcinomas; RNAi; Rac1

\section{Introduction}

Cholangiocarcinomas (CCs) are malignant tumors that originate from epithelial cells lining the biliary tree and gallbladder and grow at any location along the biliary epithelium. For practical purposes, CCs have been classified as either intrahepatic or extrahepatic, with extrahepatic sites further classified as proximal, mid, or distal (1). Intrahepatic CCs (ICCs) grow within the liver, while extra-hepatic CCs (ECCs) originate from the bile duct along the hepatoduodenal ligament. ICCs usually present as masses in the liver, while jaundice is the most common presentation of ECCs (2). CCs are relatively rare tumors, although their incidence is rising worldwide. Several advances in the diagnosis, therapy, and palliative treatment of patients affected with CCs have occurred during the last few decades (3).

The Rho family of small guanosine triphosphatases (GTPases) is a subgroup of the Ras superfamily of GTPases. In this family, Rac1, one of the most extensively studied members, was initially discovered as Ras-related C3 botulinum toxin substrate 1 in 1989 (2). The small GTPase Rac1 is a critical mediator of various aspects of endothelial cell functions (4). Rac1 signaling can affect cell growth through a variety of mechanisms. In addition to its effects on gene expression, Rac1 regulates the production of reactive oxygen species, endothelial permeability and cell adhesion, all of which have been implicated in the deterioration of neurovascular integrity in neurological diseases (3). Recent studies have implicated aberrant Rac1 activity not only in tumorigenesis, but also in neurodegenerative disorders, mental retardation syndromes, cirrhosis of the liver, and cardio-remodeling/hypertension. Rac1 expression levels have been found to be higher than normal in CCs as well as in stomach, breast, and lung cancers (3).

The intracellular signaling roles of Rac1 GTPase have been demonstrated in a body of work carried out in the past two decades (4). It is now established that Rac1, together with other Rho proteins, control the organization of actin cytoskeleton and microtubule dynamics by direct interaction with multiple effector proteins (3). It has also been revealed that Rac1 plays critical roles in integrating signals from extracellular stimuli to the cell nucleus, mediating serum response factor-, bcatenin-, and NF-kB-dependent transcription, and is required during $\mathrm{G} 1$ and $\mathrm{G} 2 / \mathrm{M}$ cell cycle progression (5).

Correspondence: Guangyi Wang, Department of General Surgery, First Clinical Hospital, Jilin University, Changchun, Jilin, 130021, China. Fax: +86-471-663-6949. E-mail wgymd@sina.com

Received October 1, 2010. Accepted March 25, 2011. Available online May 2, 2011. Published May 16, 2011. 
With these critical roles in cell regulation, it is not surprising that malfunction of Rac1 GTPase-controlled signaling pathways has begun to be associated with many aspects of human diseases including cancer (6). Thus, the possible therapeutic role of Rac1 may represent an important aspect of biomedical research.

At present, the main treatment for ECC is excision of the primary tumor, followed by radiotherapy and chemotherapy (6). In many cases, however, problems such as metastatic lesions and subsequent cancer recurrence are still encountered. Recently, following the development of modern molecular biology, in-depth studies have been conducted to develop new strategies of ECC treatment at the genetic level (7). In particular, RNA interference (RNAi) technology has the potential to become an effective treatment for tumors, and has established a new area of clinical therapy for ECC. In the present study, immunohistochemical staining using a streptavidin-peroxidase method for the Rac1 protein was performed on 74 ECC samples and 17 normal samples in an attempt to explore their roles in ECC. Moreover, a Rac1 targeting small interfering RNA (siRNA) was transferred into human ECC QBC939 cells to investigate its effects on cell proliferation.

\section{Material and Methods}

\section{Patients and samples}

Seventy-four ECC specimens and matched adjacent normal mucosa were obtained from the Department of Pathology, Inner Mongolia Medicine Hospital, between 2007 and 2009. None of the patients had been treated with radiotherapy or chemotherapy before surgery. Samples were taken from areas of the tumor tissue with no evidence of hemorrhage or putrescence. Samples of matching normal mucosa were collected from the surgical cutting edge, which was approximately $3-5 \mathrm{~cm}$ away from the cancerous lesion. The clinical diagnosis of all 74 patients was confirmed by histological examination of the resected surgical specimens. The CC cell line QBC939 was obtained from the Chinese Academy of Sciences.

\section{Immunohistochemistry}

Immunohistochemistry was performed as follows: tissue sections were deparaffinized and rehydrated three times for $3 \mathrm{~min}$ in xylene, three times for $2 \mathrm{~min}$ in $100 \%$ ethanol, once for $2 \mathrm{~min}$ in $95 \%$ ethanol, once for $2 \mathrm{~min}$ in $75 \%$ ethanol and, finally, twice for $1 \mathrm{~min}$ in distilled water. Antigens were recovered by heating the sections in a microwave oven for $15 \mathrm{~min}$, after which the sections were washed three times for $5 \mathrm{~min}$ with phosphate-buffered saline (PBS). Endogenous peroxidase activity was blocked by soaking the slides in a solution of $3 \%$ hydrogen peroxide for $15 \mathrm{~min}$ at room temperature, followed by washing three times for 5 min with PBS. Non-immune goat blood serum $(50 \mu \mathrm{L})$ was added to each section for $15 \mathrm{~min}$ at room temperature, followed by the primary antibodies (Millipore, $50 \mu \mathrm{L}$ ). Slides were incubated overnight at $4^{\circ} \mathrm{C}$ in a humidified chamber and washed three times for $10 \mathrm{~min}$ in PBS. Biotin-labeled secondary antibodies ( $50 \mu \mathrm{L})$ were added to each section and kept for $15 \mathrm{~min}$ at $37^{\circ} \mathrm{C}$, followed by washing three times for $5 \mathrm{~min}$ with PBS. Samples were incubated for 15 min at room temperature and washed three times for 5 min in PBS before the addition of $100 \mathrm{~L}$ freshly prepared 3,3-diaminobenzidine for approximately 5-20 min. The reaction was stopped by washing in cold water. Slides were counterstained with hematoxylin, followed by a sealing procedure using neural gum.

\section{miRNA design}

The Rac1-targeting miRNA sequence that was designed as gene bank ID NM_018890 by Invitrogen Company was sense-CACCGACTTCATAAGGCGCATGC, antisenseGCATGCCGTCTTGAAGCATGCGCCTTATGAAGTC, loop-TTCAAGACG.

\section{Cell culture}

The CC cell line QBC939 was grown as a monolayer in RPMI-1640 (Hyclone) containing 10\% fetal bovine serum. Cells were maintained at $37^{\circ} \mathrm{C}$ in a humidified incubator under a $5 \% \mathrm{CO}_{2}$ atmosphere.

\section{Transfection of miRNA}

miRNA transfection was performed according to the protocol supplied by the Invitrogen Company. Briefly, $1 \times 10^{5}$ cells were seeded into six-well plates containing an antibiotic-free medium and incubated overnight. For each well, $5 \mu \mathrm{L}$ miRNA was mixed with $125 \mu \mathrm{L}$ OPTI-MEM I. The mixture was then combined with a solution of $5 \mu \mathrm{L}$ lipofectamine (Invitrogen Company) in $125 \mu \mathrm{L}$ OPTI-MEM I. After a 20 -min incubation period at room temperature, the mixture was applied to the cells in an appropriate volume of OPTI-MEM I so as to achieve a final concentration of $100 \mathrm{nM}$ for each miRNA. After incubation for $6 \mathrm{~h}$ at $37^{\circ} \mathrm{C}$, RPMI-1640 supplemented with serum was added to the wells and cells were cultured for an additional $24 \mathrm{~h}$ at $37^{\circ} \mathrm{C}$ before analysis.

\section{Western blotting}

Cells were harvested $48 \mathrm{~h}$ after transfection, washed with cold PBS, and lysed with a phenylmethanesulfonyl fluoride lysis buffer. After centrifugation at 13,200 $\mathrm{g}$ for 30 min, the supernatant was analyzed for protein content using the bicinchoninic acid reagent. Total proteins $(50 \mu \mathrm{g})$ from each sample were electrophoresed on a $10 \%$ SDS-PAGE gel and transferred to a polyvinylidene fluoride membrane. The membranes were blocked with PBS containing 5\% fat-free milk and $0.1 \%$ Tween-20 for 30 min at room temperature, and then incubated with primary anti-Rac1 antibody for at least $1 \mathrm{~h}$ at room temperature, or overnight at $4^{\circ} \mathrm{C}$. The membranes were washed three times with PBS containing $0.1 \%$ Tween 20 , incubated with peroxidase- 
conjugated secondary antibodies, and developed using the ECL reagent (Pierce).

\section{3-(4,5-Dimethyldiazol-2-yl)-2,5-diphenyl tetrazolium bromide (MTT) assay}

Cells were grown in 96-well plates $\left(1 \times 10^{3}\right.$ cells/well) and transfected with Rac1-targeting miRNA. Control cells were switched from RPMI-1640 to DMEM containing $0.1 \%$ dimethyl sulfoxide (DMSO). At 24, 48, and $72 \mathrm{~h}$ following miRNA transfection, $20 \mu \mathrm{L}$ MTT was added to each well to a final concentration of $0.5 \%$. After a $4-\mathrm{h}$ incubation at $37^{\circ} \mathrm{C}$ in the dark, $150 \mu \mathrm{L}$ DMSO was added to each well for 10 min to dissolve the formazan crystals. Absorbance at $490 \mathrm{~nm}$ was measured using a microplate reader (EXL800, USA). All experiments were repeated three times. The viability of the miRNA-transfected cells is reported as percent population growth plus the standard error of the mean relative to that of untransfected control cells. Cell death caused by miRNAtransfection was calculated as percent inhibition as follows: $\%$ inhibition $=(1$ - mean experimental absorbance $/$ mean control absorbance) $\times 100$.

\section{Flow cytometry analysis}

At $48 \mathrm{~h}$ after transfection, samples $\left(1 \times 10^{6}\right.$ cells $)$ were
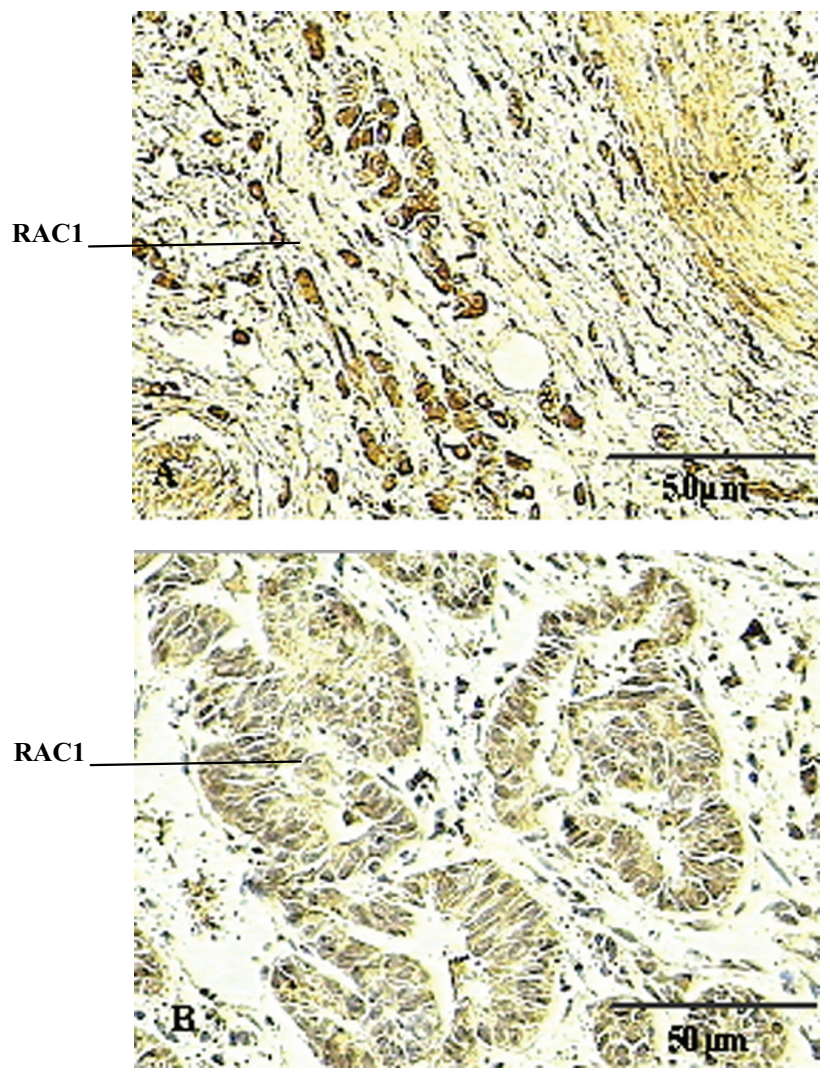

fixed in $70 \%(\mathrm{v} / \mathrm{v})$ ethanol and washed, and RNAse $(0.25$ $\mathrm{mg} / \mathrm{mL})$ and Tween $20(0.1 \%(\mathrm{v} / \mathrm{v}))$ were added for $30 \mathrm{~min}$ at room temperature. The cells were stained with propidium iodide and analyzed using a flow cytometer (EPICS XL; Beckman). Several peaks, including sub-G0-G1, S, and G2/M peaks, were detected on the DNA plots using the Cell Quest software. The sub-G0-G1 peak was assumed to indicate the rate of cell apoptosis.

\section{Statistical analysis}

The statistical software package SPSS 13.0 was used and data were analyzed by the $\mathrm{X}^{2}$ test or the Fisher exact test. Non-parametric Spearman rank correlation analysis was used to analyze ranked data. The rates were compared using $Z$ tests. A P value $<0.05$ was regarded as significant.

\section{Results}

\section{Expression of the Rac1 protein in ECC}

Of the 74 ECC cases analyzed, $17(22.97 \%)$ were highly differentiated adenocarcinomas, 29 (39.19\%) moderately differentiated adenocarcinomas, and 28 (37.84\%) slightly differentiated adenocarcinomas. In all 17 normal cases, expression of Rac1 was relatively low (Figure 1). On the other

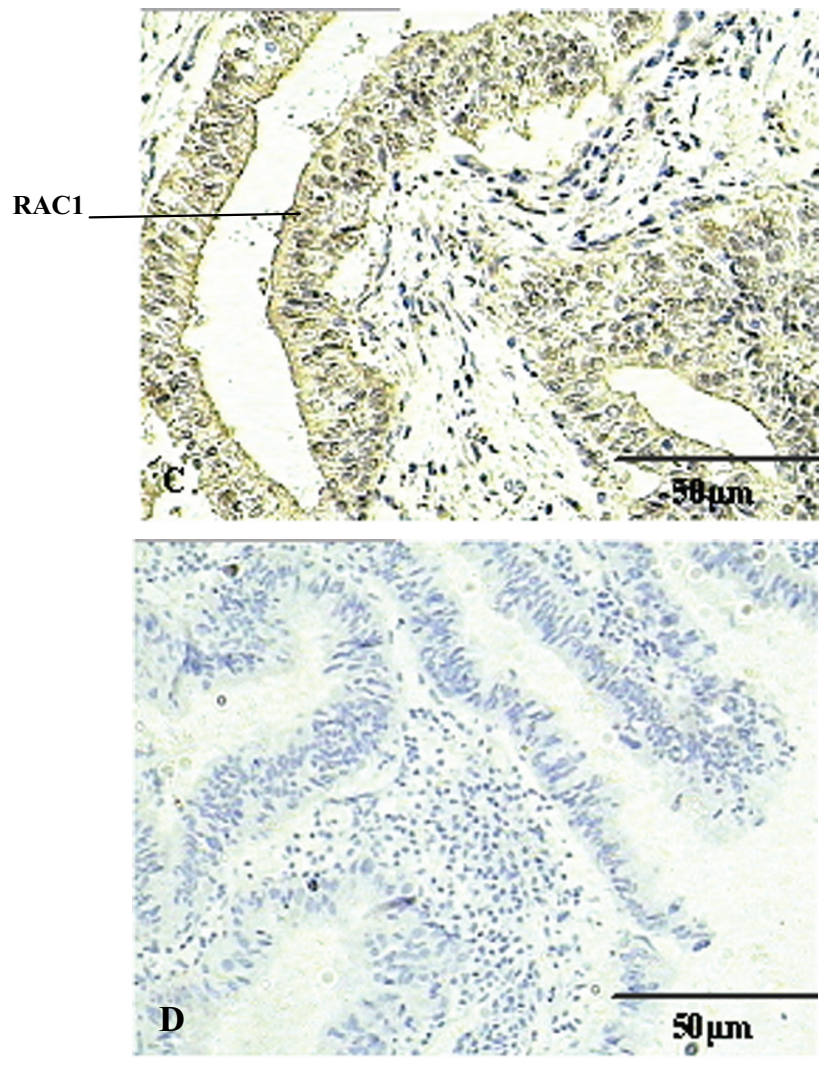

Figure 1. Expression of the Rac1 protein (arrows) in extrahepatic cholangiocarcinomas. $A$, Slightly differentiated adenocarcinomas; $B$, moderately differentiated adenocarcinomas; $C$, highly differentiated adenocarcinomas; $D$, negative control. 
hand, in the three types of differentiated adenocarcinomas, Rac1 expression was substantially higher compared to normal cases. These results confirm that the Rac1 protein is present at significantly higher levels in ICC cases than in normal mucosa $(P<0.01)$.

\section{Expression of the Rac1 protein in QBC939 cells after transfection with miRNA}

The cells positively stained by the anti-Rac1 antibody accounted for $95-99 \%$ of the total cell population. The control group showed strongly positive cytoplasmic expression of Rac1 (dark brown-yellow), while the Rac1-miRNA group showed weakly positive cytoplasmic expression (light brown-yellow). Western blotting showed that the Rac1 miRNA-transfected cells expressed significantly less Rac1 than the control groups (Figure 2).

\section{Effect of miRNA on cell proliferation}

The proliferation rate of the QBC939 cell line 24, 48, and $72 \mathrm{~h}$ after transfection with Rac1-miRNA was significantly higher than that of the control group, as assessed by the MTT assay (Figure 3). These effects were time-dependent and clearly showed that cell proliferation was markedly inhibited in the Rac1-miRNA-transfected group.

\section{Cell apoptosis detected by flow cytometry}

To test for apoptotic cells, we used flow cytometry (FCM) to locate apoptotic sub-G0-G1 (M1 value), S, and $\mathrm{G} 2 / \mathrm{M}$ peaks $48 \mathrm{~h}$ after miRNA transfection. The results showed that, compared to the control group $(\mathrm{M} 1=0.59)$ and the mismatched group (M1 = 0.63), the Rac1-miRNA group displayed an apparent aneuploid peak (M1 = 13.88; Figure 4), thus suggesting ongoing apoptosis in the cells transfected with Rac1-miRNA.

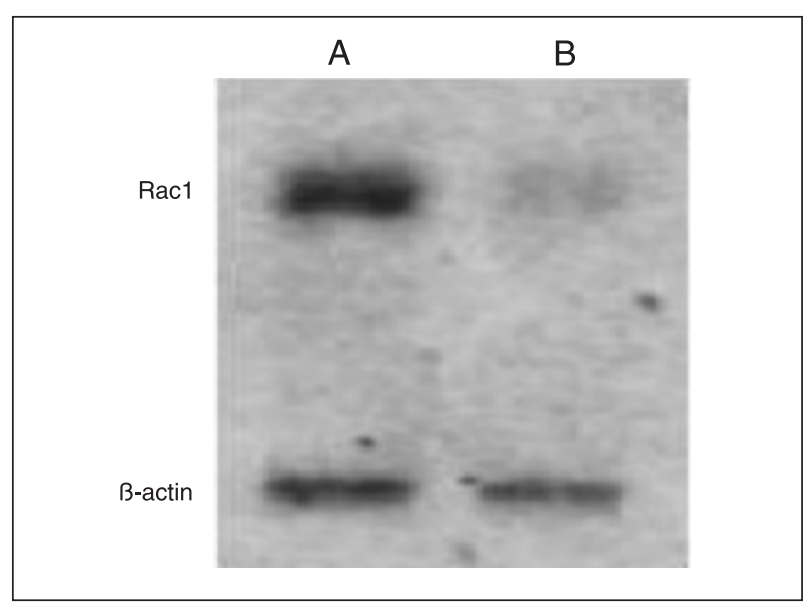

Figure 2. Expression of Rac1 in QBC939 cells after Rac1-interfering RNA (miRNA) transfection. Lane A, Control group; Lane B, Rac1-miRNA group.

\section{Discussion}

$\mathrm{CC}$ is the second most common type of liver cancer after hepatocellular carcinoma (7). Biliary tract cancers are challenging to diagnose and treat and chronic inflammation and obstruction of bile ducts may play an important role in the progression of this deadly disease (8). Once diagnosed, $\mathrm{CC}$ is commonly treated by surgical resection; however, this option is not always viable (9). Although Rho GTPases have been shown to be required for cellular functions associated with tumor progression and invasiveness, the role of Rho proteins in carcinogenesis and tumor progression in actual patients with cancer remains unconfirmed $(7,8)$. Rac GTPases play significant roles in various cellular processes, including migration, cytokinesis, lamellipodia formation, and cell polarity (3). Different studies have indicated contradictory roles for Rho proteins in tumor progression $(10,11)$. For example, although Rac1 down-regulation was shown to result in the inhibition of migration in most human malignancies, migration was shown to be stimulated in aggressive breast cancer cells where Rac1 was knocked down (7). Rac1 positively regulates transcription at NF-kB transcription factor-dependent promoters and facilitates phosphatidylinositol-3 kinase-dependent activation of AKT ser/thr kinase, thereby permitting the survival of transformed cells $(2,9)$. Rac1 can also influence transformation by regulation of cyclin $\mathrm{D} 1$, a cell cycle protein that is frequently overexpressed in cancer (12). High RhoA activities were related to the poor prognosis of several cancers (10), such as liver, esophagus, testicular, and upper urinary tract cancers, as well as ovarian carcinoma, whereas high expression of RhoA indicated favorable outcomes in colorectal cancers and PDAC (13). In addition to tumor types, other factors may also influence how Rho proteins affect cell behaviors.

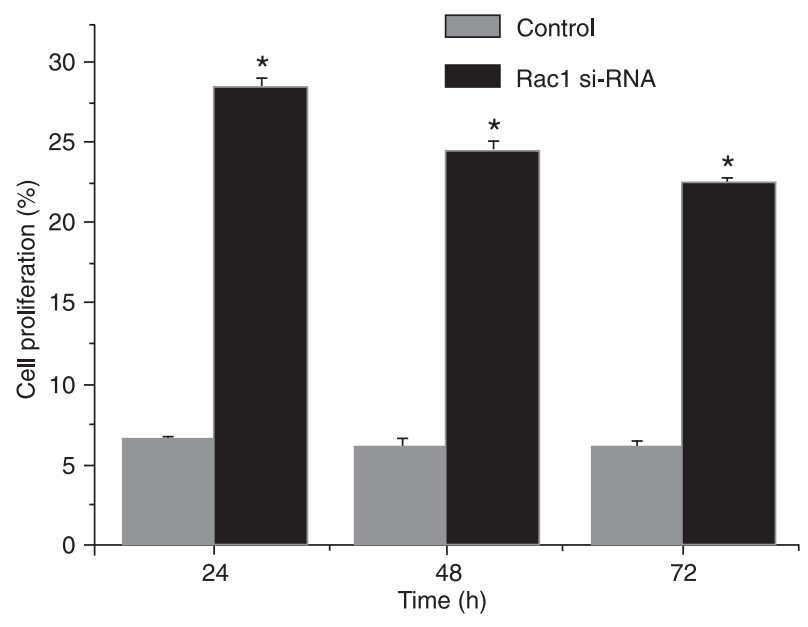

Figure 3. Effect of miRNA on cell proliferation as determined by the 3-(4,5-dimethyldiazol-2-yl)-2,5-diphenyl tetrazolium bromide (MTT) assay. Data are reported as means \pm SEM. ${ }^{*} \mathrm{P}<0.05 \mathrm{com}-$ pared to control ( $t$-test). 

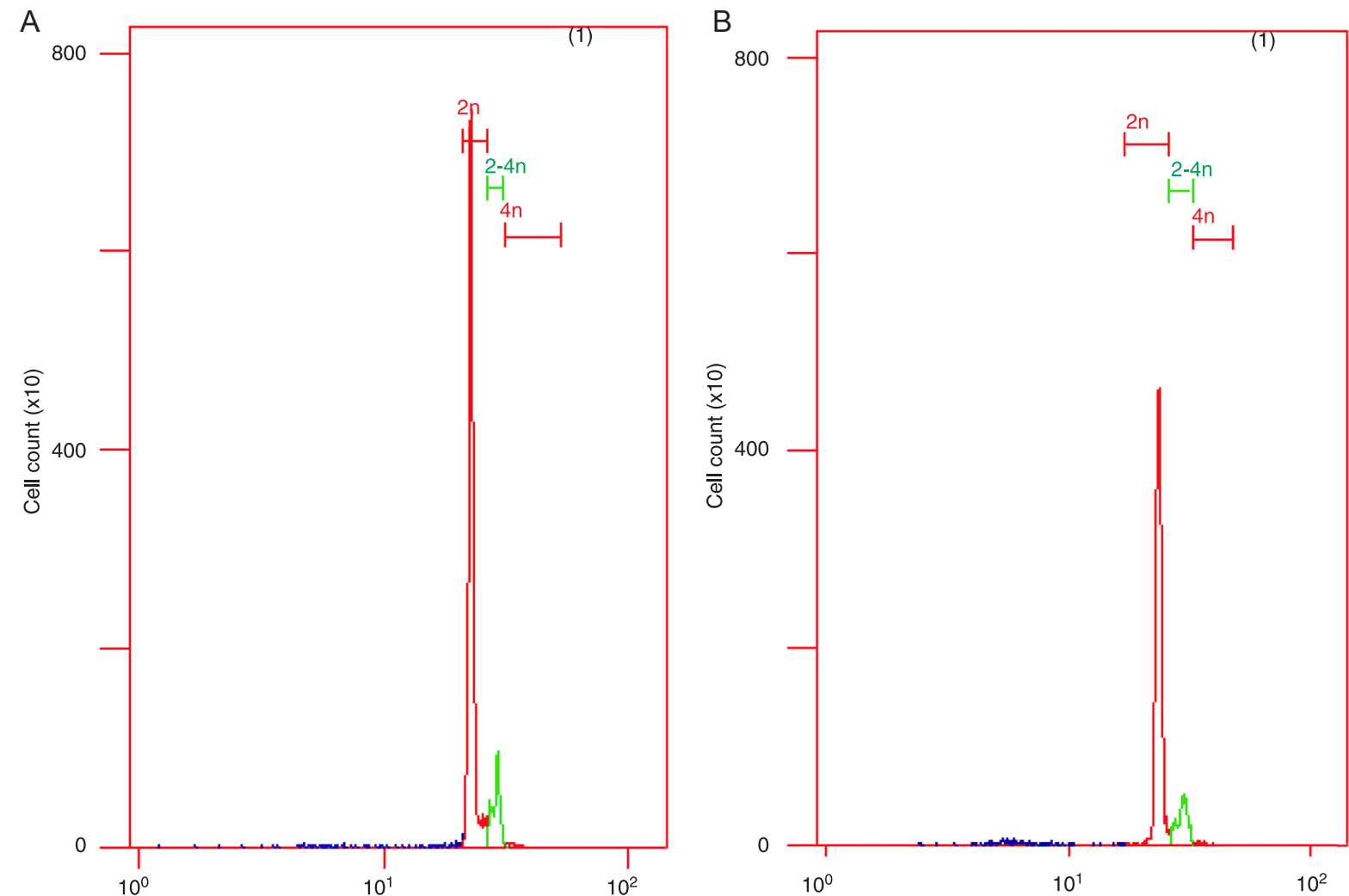

Figure 4. A, Apoptosis of QBC939 cells measured by flow cytometry. Control group (left); Rac1-miRNA group (right). B, QBC939 cell apoptosis detected by flow cytometry after transfection. Data are reported as means \pm SEM. ${ }^{*} \mathrm{P}<0.05$ compared to control $(t$-test).

For example, the extracellular matrix can change the effect of Rac1 in promoting cell-cell adhesion (2). Additionally, Rac1 was colocalized with MMP-9 in CCs, and it may influence the DNA damage induced by cholangiocarcinogenesis (11). In the present study, Rac1 was able to inhibit the growth of ECC cells. The ECC was greatly disrupted when high Rac1 levels were expressed. This is consistent with previous studies reporting that Rac1 can inhibit colitis-related CC progression (12).

RNAi is an important technique that specifically downregulates the expression of cellular genes and has opened the way to the therapeutic use of siRNAs. In our study, we used RNAi technology to transfect an siRNA targeted against Rac1 into QBC939 cells using Lipofectamine 2000. Western blotting showed that the expression of the Rac1 protein was significantly reduced. Our results indicate that Rac1-siRNAcan actively suppress Rac1 protein expression and inhibit cell proliferation, in agreement with current literature $(14,15)$. Blocking Rac1 expression using RNAi resulted in significant inhibition of cell growth in a time-dependent manner. A recent study (14) showed that Rac1 is closely associated with apoptosis. Lin and van Golen (16) found that Rac1 could suppress apoptosis in breast cancer cells. Aznar and Lacal (17) found that Rac1 could up-regulate bcl-2 levels in breast cancer cells, thus inhibiting apoptosis in both tumor cells and vascular endothelial cells. Our results show that the rate of apoptosis was significantly higher in the Rac1-siRNA group than that in the control group. This suggests that siRNAcan effectively silence the Rac1 gene and promote apoptosis in QBC939 cells.

Rac1 can promote cytoskeleton construction, intercellular sticking, and intercellular signal transmission, as well as cell proliferation. Rac1 can also increase NAPDH levels and protect cells from apoptosis (13). In the present study, the slightly differentiated adenocarcinoma group had the highest Rac1 expression level and in QBC939 cells, the RNAi group showed more apoptosis than the control group.

Taken together, the present results suggest that Rac1 is associated with CC carcinogenesis. Blocking Rac1 expression using RNAi technology significantly reduced Rac1 protein levels, suppressed the proliferation of QBC939 cells, and induced apoptosis. Our data thus indicate that the use of miRNA targeting Rac1 may have a therapeutic potential for the treatment of ECCs.

\section{Acknowledgments}

Research supported by the Inner Mongolia Natural Science Foundation (\#NM 006908), The People's Republic of China. 


\section{References}

1. Hernandez J, Cowgill SM, Al-Saadi S, Villadolid D, Ross S, Kraemer E, et al. An aggressive approach to extrahepatic cholangiocarcinomas is warranted: margin status does not impact survival after resection. Ann Surg Oncol 2008; 15: 807-814

2. Bosco EE, Mulloy JC, Zheng Y. Rac1 GTPase: a "Rac" of all trades. Cell Mol Life Sci 2009; 66: 370-374.

3. Ji P, Lodish HF. Rac GTPases play multiple roles in erythropoiesis. Haematologica 2010; 95: 2-4.

4. Lubell J. More changes on the RAC. Mod Healthc 2010; 40: 29

5. Guo D, Zhang JJ, Huang XY. A new Rac/PAK/GC/cGMP signaling pathway. Mol Cell Biochem 2010; 334: 99-103.

6. Schlegel N, Waschke J. Impaired cAMP and Rac 1 signaling contribute to TNF-alpha-induced endothelial barrier breakdown in microvascular endothelium. Microcirculation 2009; 16: 521-533.

7. Davis RL. Rac in the act of forgetting. Cell 2010; 140: 456458.

8. Shuai Y, Lu B, Hu Y, Wang L, Sun K, Zhong Y. Forgetting is regulated through Rac activity in Drosophila. Cell 2010; 140: 579-589.

9. Safian SC. The RAC coordinator's role. RAC audits require a coordinator to ensure compliance. J AHIMA 2009; 80: 49.

10. Wu YI, Frey D, Lungu OI, Jaehrig A, Schlichting I, Kuhlman $B$, et al. A genetically encoded photoactivatable Rac controls the motility of living cells. Nature 2009; 461: 104-108.

11. Prakobwong $S$, Yongvanit $P$, Hiraku $Y$, Pairojkul $C$, Sithithaworn $\mathrm{P}$, Pinlaor $\mathrm{P}$, et al. Involvement of MMP-9 in peribiliary fibrosis and cholangiocarcinogenesis via Rac1dependent DNA damage in a hamster model. Int $\mathrm{J}$ Cancer 2010; 127: 2576-2587.

12. Wang Z, Jin H, Xu R, Mei Q, Fan D. Triptolide downregulates Rac1 and the JAK/STAT3 pathway and inhibits colitis-related colon cancer progression. Exp Mol Med 2009; 41: 717727.

13. Eklund DM, Svensson EM, Kost B. Physcomitrella patens: a model to investigate the role of RAC/ROP GTPase signalling in tip growth. J Exp Bot 2010; 61: 1917-1937.

14. Sanlioglu S, Luleci G, Thomas KW. Simultaneous inhibition of Racl and IKK pathways sensitizes lung cancer cells to TNFalpha-mediated apoptosis. Cancer Gene Ther 2001; 11: 897-905.

15. Joneson T, Bar-Sagi D. Suppression of Ras-induced apoptosis by the Rac GTPase. Mol Cell Biol 1999; 19: 58925901.

16. Lin $\mathrm{M}$, van Golen KL. Rho-regulatory proteins in breast cancer cell motility and invasion. Breast Cancer Res Treat 2004; 84: 49-60.

17. Aznar S, Lacal JC. Rho signals to cell growth and apoptosis. Cancer Lett 2001; 165: 1-10. 